\title{
CURVAS DE ABSORCIÓN DE NUTRIMENTOS PARA TRES VARIEDADES DE LIRIOS (Lilium sp.) Y AFINAMIENTO DEL PROGRAMA DE FERTILIZACIÓN EN UNA FINCA COMERCIAL EN HEREDIA, COSTA RICA
}

\author{
Bianca L. Barrantes-Infante ${ }^{1 / *}$, Floria Bertsch ${ }^{*}$
}

\begin{abstract}
Palabras clave: Lirios, bulbo, absorción, fertilización, nutrición.
\end{abstract} Keywords: Lilies, bulb, absorption, fertilization, nutrition.

\section{RESUMEN}

A partir de la obtención de las curvas de absorción de nutrimentos de las variedades de lirio Brunello, Algarve y Alma Ata, se evaluó y afinó el programa de fertilización en una finca comercial. Se evaluó el peso fresco y seco a $80^{\circ} \mathrm{C}$ cada 15 días, de 3 plantas por variedad, separadas por órgano. A los tejidos secos se les realizó análisis de concentración y a partir de estos datos se obtuvo la absorción de nutrimentos. Con base en las curvas de absorción se establecieron 5 programas de fertilización: Finca, Requisitos, Ajustado, Sin Granular y Testigo Absoluto; los cuales se aplicaron a plantas de lirio variedad Siberia, durante un ciclo de crecimiento. Las curvas indicaron que en las primeras 4 semanas de un ciclo total de 12 a 14 semanas, la mayor parte de los nutrimentos que utiliza la planta provienen del bulbo. Los contenidos de nutrientes de N, P y K en los bulbos al momento de la siembra oscilaron entre 144-178, 19-28 y 160-174 mg.bulbo ${ }^{-1}$, respectivamente; mientras que para una producción de 66 (de Alma Ata) y 72 (de Brunello y Algarve) tallos. $\mathrm{m}^{-2}$ el consumo por la parte área fluctuó entre 15-18; 1,2-1,6 y 26,7-40,3 g. $\mathrm{m}^{-2}$, respectivamente. En todas las variedades, la mayor absorción de estos nutrimentos ocurrió al final del período de vida. Con el programa de Requisitos se logró mantener, sin diferencias

1 Autor para correspondencia. Correo electrónico: bbarrantes@gmail.com

\section{ABSTRACT}

Nutrient absorption curves in three lily (Lillium sp.) varieties and optimization of a fertilization program in a commercial farm in Heredia, Costa Rica. The fertilizer program was evaluated and optimized in a lily commercial farm, after the development of nutrient absorption curves in the varieties Brunello, Algarve and Alma Ata. The lily plants were separated into organs to evaluate the fresh and dry at $80^{\circ} \mathrm{C}$ weights, every 15 days, in 3 plants per variety. Dry tissues underwent concentration analysis to obtain the nutrient absorption. Based on nutrient absorption curves 5 fertilization treatments were designed: a treatment that consisted in the fertilization program of the commercial farm; the requirement treatment; which included only what the plant absorbs; an adjusted treatment, a treatment without any granular application; and the control. These treatments were applied to the Siberia variety during the growing cycle. The absorption curves showed that most of the nutrients are provided by the bulb during the first 4 weeks of a total cycle of 12 to 14 weeks. The contents of N, P and $\mathrm{K}$ in the bulbs at the moment of planting ranged from 144-178, 19-28 and 160$174 \mathrm{mg} . \mathrm{bulb}^{-1}$, respectively, while for a production of 66 (Alma Ata) and 72 (Brunello and Algarve) shoots. $\mathrm{m}^{-2}$ the aerial part of the plant consumed

\footnotetext{
* Centro de Investigaciones Agronómicas, Universidad de Costa Rica.
} 
significativas, el nivel de producción y calidad de la finca, además se obtuvo el mayor número de plantas de tamaños medianos y grandes con valor comercial. Las plantas de los programas Requisitos y Ajustado mostraron el mayor número de botones de tamaño grande $(>5 \mathrm{~cm})$. Las aplicaciones foliares demostraron ser importantes, ya que el programa Sin Granular presentó el mayor porcentaje (56\%) de bulbos y raíces adventicias con la mejor apariencia.

\section{INTRODUCCIÓN}

En Costa Rica, las primeras plantaciones de flores se establecieron hace 35 años, por extranjeros radicados en el país. Entre 1982 y 1985 se dio el incremento de las áreas de siembra de flores de corta, debido al estímulo del gobierno a las actividades de exportación (A. Durán. 2008. Producción comercial de flores en Costa Rica. Comunicación personal). Entre el 2003 al 2007 las exportaciones de flores y capullos fluctuaron desde los \$29 millones hasta los \$42 millones, lo que aumentó las cifras año tras año (PROCOMER 2004-2008); no obstante a partir del 2008 estas cifras se redujeron a $\$ 37$ millones (Arce et al. 2008), en el 2009 a $\$ 33$ millones (Chaves et al. 2009), y en octubre del 2010 esta cifra había disminuido a \$28 millones (PROCOMER 2010), lo cual posiblemente se deba a la crisis económica mundial, que hizo que los compradores, especialmente los de Estados Unidos (principal destino de las exportaciones), redujeran sus gastos en los artículos no básicos, como lo son las flores.

En el mercado de la floricultura, las plantas de bulbo como el tulipán y los lirios (Facchinetti
15-18, 1.2-1.6 and 26.7-40.3 g.m ${ }^{-2}$, respectively. In all varieties, the highest absorption of $\mathrm{N}, \mathrm{P}$ and $\mathrm{K}$ occurred at the end of the life cycle. The requirement treatment did not show significant differences from the treatment of the commercial farm, presenting the same level of production and quality; also, it produced the highest number of medium and large size plant with a commercial value. The requirement and adjusted treatments showed the highest number of large buttons $(>5 \mathrm{~cm})$. Foliar applications of fertilizers proved to be important, mainly because the treatment without any granular fertilizer application had the highest percentage (56\%) of bulbs and adventitious roots with the best appearance.

y Marinangeli 2008) son las de mayor importancia; su producción abarca una superficie de 31 000 ha en el mundo, donde Holanda es el país que engloba el 65\% de la producción total, con 10000 millones de bulbos, entre ellos tulipanes, lirios, jacintos y narcisos.

Para el 2007 y 2008, en Costa Rica, las exportaciones de lirios abarcaron el $52 \%$ y $50 \%$, respectivamente, de las exportaciones de flores y capullos (Hernández 2008, Arce 2009).

A pesar de la importancia que las flores de lirios han tenido en el mercado de la floricultura mundial, en la actualidad existe muy poca información sobre programas de fertilización específicos en este cultivo. Para el caso de Lilium sp., existe una gran variabilidad genética, por lo que las recomendaciones deben tomar en cuenta la variedad (Ortega et al. 2006).

Es por esto que el objetivo de este trabajo fue obtener las curvas de absorción de nutrimentos de al menos 3 variedades de lirio (Brunello, Algarve y Alma Ata) para conocer su variabilidad, y apoyándose en ellas proponer mejoras en un programa de fertilización de una finca comercial de lirios. 


\section{MATERIALES Y MÉTODOS}

\section{Localización}

El trabajo se llevó a cabo en la empresa Flor Bella S.A., ubicada en San José de la Montaña, Heredia, Costa Rica, a 1807 msnm. Los análisis foliares y de suelos se realizaron en el Laboratorio de Suelos y Foliares (LSF) del Centro de Investigaciones Agronómicas (CIA), de la Universidad de Costa Rica.

\section{Material experimental}

Las variedades de lirios utilizadas en este trabajo pertenecen al tipo de lirio llamado Lilium de color. Se trabajó con las variedades Brunello, Algarve, Alma Ata y Siberia, pertenecientes a los grupos Asiático, Híbrido L/A y Oriental, las 2 últimas, respectivamente, con ciclos de vida de 10, 10, 14 y 14 semanas, respectivamente. Los bulbos utilizados provenían de Holanda y solo se utilizaron una vez.

\section{Curvas de absorción}

Las curvas de absorción se realizaron sobre 3 variedades de lirio (Brunello, Algarve, Alma Ata) en una plantación bajo invernadero establecida por la finca, a partir de bulbos con un calibre de 12/14 (tamaño del bulbo). Las evaluaciones se realizaron entre agosto y octubre, cada 15 días, a partir del primer mes posterior a la siembra, hasta un día antes de la cosecha. En cada etapa se evaluaron 2 tejidos, parte aérea con flor y bulbo + raíces adventicias. La finca utilizaba riego por aspersión.

Se realizaron en total 4 evaluaciones, para el caso de las variedades Brunello y Algarve (con ciclo de vida de 10 semanas) y 6 evaluaciones para la variedad Alma Ata (con ciclo de vida de 14 semanas). En cada evaluación se tomaron 3 plantas por variedad para un total de 12 plantas (para las variedades de tipo Asiático y L/A) y 18 plantas (para las variedad tipo Oriental).

Las plantas fueron separadas por órgano (parte aérea y bulbo + raíces adventicias) y se les tomó el peso fresco y seco a $80^{\circ} \mathrm{C}$ aproximadamente a cada parte, para luego ser analizadas según el procedimiento de rutina en el Laboratorio de Suelos y Foliares de la Universidad de Costa Rica, que consiste en la digestión total de la muestra con ácido nítrico y la determinación de los elementos con el Espectrofotómetro de Emisión Atómica con Plasma. El N se determinó por combustión seca en un autoanalizador.

Al asociar los valores de materia seca (g.planta ${ }^{-1}$ ) con la concentración de los distintos elementos que se analizaron en cada muestra, se calculó la absorción (g.planta ${ }^{-1}$ ) de los nutrimentos y se elaboraron las curvas de absorción (Bertsch 2003).

Un resumen del estado del suelo en los diferentes lotes usados para muestrear las diferentes edades de las plantas se presentan en el Cuadro 1. Para todas las características, el suelo mostró condiciones apropiadas.

Cuadro 1. Ámbito de análisis de suelos presente en los lotes muestreados, Finca Flor Bella S.A., Heredia, Costa Rica.

\begin{tabular}{cccccccccccc}
\hline $\mathrm{pH}$ & & \multicolumn{3}{c}{$\mathrm{cmol}(+) .1^{-1}$} & & \multicolumn{4}{c}{$\%$} & \multicolumn{4}{c}{$\mathrm{mg} \cdot \mathrm{l}^{-1}$} \\
\hline $\mathrm{H}_{2} \mathrm{O}$ & $\mathrm{Acidez}$ & $\mathrm{Ca}$ & $\mathrm{Mg}$ & $\mathrm{K}$ & $\mathrm{CICE}$ & $\mathrm{SA}$ & $\mathrm{P}$ & $\mathrm{Zn}$ & $\mathrm{Cu}$ & $\mathrm{Fe}$ & $\mathrm{Mn}$ \\
\hline $5,3-5,8$ & $0,08-0,13$ & $6,88-10,50$ & $2,37-3,40$ & $0,79-1,08$ & $8,48-14,48$ & $<1 \%$ & $11-36$ & $5,6-18,0$ & $4-7$ & $22-31$ & $9-17$ \\
\hline
\end{tabular}




\section{Programa de fertilización}

Con base en las curvas de absorción de nutrimentos se diseñaron 2 programas de fertilización con el fin de buscar si estos resultaban mejores alternativas que los que se usaban en la finca: uno llamado Programa de Requisitos, el cual se basó en la utilización estricta de los requisitos de nutrimentos establecidos según la curva; y el otro llamado Programa Ajustado que consistió en el uso de la mitad de los requisitos, ya que se consideró que el suelo podría participar en el suplemento de nutrimentos también.
Solo en el caso del P se tomaron criterios particulares en este tratamiento que serán discutidos más adelante.

Paralelamente se establecieron otros 3 tratamientos: Programa Sin Granular, en el que se probó la capacidad de suplemento total del suelo, por medio solo de las aplicaciones foliares rutinarias en la finca; el Programa propio de la Finca, y un Testigo Absoluto. Los fertilizantes granulares utilizados en cada uno de los programas fueron aplicados al voleo y en el Cuadro 2 se especifican los productos y dosis utilizados en los programas de fertilización.

Cuadro 2. Productos y dosis utilizados en los programas de fertilización Finca, Requisitos y Ajustados, en plantas de lirio tipo Oriental, en San José de la Montaña, Heredia.

\begin{tabular}{|c|c|c|}
\hline Programa & Producto & Dosis g. $\mathrm{m}^{-2}$ \\
\hline Finca & $\begin{array}{l}\text { 12-24-12 } \\
\text { Nitrato de Calcio } \\
\text { Sulfato de Magnesio } \\
\text { Magnesamón }\end{array}$ & $\begin{array}{l}50 \\
12 \\
10 \\
6\end{array}$ \\
\hline Requisitos & $\begin{array}{l}\text { 12-24-12 } \\
\text { Nitrato de Calcio } \\
\text { Nitrato de Potasio } \\
\text { Kmag }\end{array}$ & $\begin{array}{l}9 \\
23 \\
59 \\
20\end{array}$ \\
\hline Ajustado & $\begin{array}{c}\text { DAP } \\
\text { Nitrato de Calcio } \\
\text { Nitrato de Potasio } \\
\text { Kmag } \\
\text { Sulfato de Potasio }\end{array}$ & $\begin{array}{l}14 \\
12 \\
12 \\
11 \\
16\end{array}$ \\
\hline
\end{tabular}

A todos los programas, excepto al Testigo Absoluto, se les apoyó con las aplicaciones foliares regulares realizadas por la empresa Flor Bella S.A., que incluía productos como Crop-up $(0,8$ ml.m $\left.{ }^{-2}\right)$, NPK $\left(4,5 \mathrm{ml} \cdot \mathrm{m}^{-2}\right)$, Pholex Fe $\left(6,5 \mathrm{ml} \cdot \mathrm{m}^{-2}\right)$, Molibdato de $\mathrm{Na}\left(0,2 \mathrm{ml} . \mathrm{m}^{-2}\right)$, y Metalosatos de $\mathrm{Ca}\left(4,5 \mathrm{ml} \cdot \mathrm{m}^{-2}\right), \mathrm{Mg}\left(3 \mathrm{ml} \cdot \mathrm{m}^{-2}\right), \mathrm{B}\left(0,6 \mathrm{ml} \cdot \mathrm{m}^{-2}\right)$, $\mathrm{Cu}\left(0,9 \mathrm{ml} \cdot \mathrm{m}^{-2}\right), \mathrm{Mn}\left(1,8 \mathrm{ml} \cdot \mathrm{m}^{-2}\right)$ y $\mathrm{Zn}\left(3 \mathrm{ml} \cdot \mathrm{m}^{-2}\right)$. 
Cada programa constó de 3 repeticiones y cada repetición estuvo conformada por 4 cajas, con 13 plantas cada caja. El diseño experimental utilizado fue de Bloques Completos al Azar.

Una vez aplicados los respectivos programas de fertilización y llegado el momento de cosecha, se procedió a realizar las evaluaciones respectivas. En primer lugar, se tomaron todas las plantas de cada repetición y tratamiento, y se dividieron por tamaño en 3 grupos: grandes (mayor a $90 \mathrm{~cm}$ ), medianas $(80-90 \mathrm{~cm})$ y pequeñas (menor a $80 \mathrm{~cm}$ ), tomando esto como una variable de evaluación. Se realizó este agrupamiento para facilitar la evaluación. Una vez agrupadas las plantas, a las mismas se les evaluó, de manera individual, el número y peso fresco por planta, así como el número y peso fresco de botones florales según el tamaño de planta. Posteriormente, los botones florales de cada tratamiento fueron divididos por tamaño, y a cada grupo se les evaluó el número, peso fresco y seco. Otra de las variables evaluadas fue la apariencia de los bulbos y raíces, lo cual se realizó al clasificar el aspecto de los mismos, en mala, media y buena condición.

Para evaluar estadísticamente si los programas de fertilización propuestos ofrecen respuestas distintas al programa establecido por la empresa, se utilizó el programa estadístico Infostat, en donde se realizó una prueba de comparación de medias, con la prueba LSD, en donde se compararon las diferentes variables evaluadas.

\section{RESULTADOS Y DISCUSIÓN}

\section{Acumulación de peso seco}

En la Figura 1 se presenta el acúmulo de peso seco en el tiempo, efectuado por cada uno de los tejidos de plantas de las 3 variedades de lirio en estudio.

Como es posible observar, el peso seco de los bulbos durante las primeras semanas fue mayor que el peso seco de la parte aérea, lo cual se debe al alto contenido de nutrimentos que como órgano de reserva, presenta el bulbo al inicio del cultivo y que además según Betancourt et
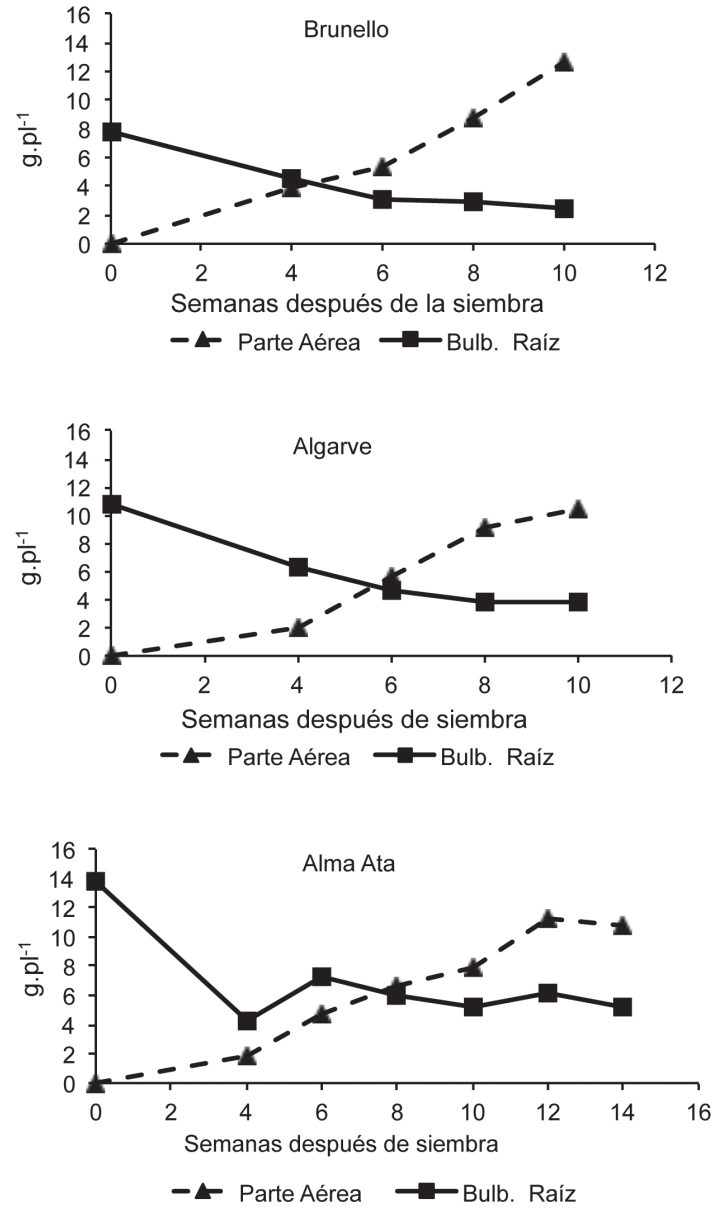

Fig. 1. Peso seco acumulado a través del tiempo por la parte aérea y el bulbo de las variedades de lirios Brunello, Algarve y Alma Ata, en San José de la Montaña, Heredia.

al. (2005), tiene la función de completar el ciclo de vida de las plantas de lirios sin necesidad de aportes nutricionales, solo basándose en lo que éste órgano les suministra.

Asimismo, se observa que con el paso de las semanas, el peso seco de los bulbos disminuyó, mientras que el de la parte aérea fue en aumento, debido a la translocación de nutrimentos que ocurre desde el bulbo hacia la parte aérea (Miller, citado por Correa 2003), lo cual se explica por la 
alta correlación entre la concentración inicial de almidón en el bulbo y el peso seco del mismo (Toit et al. 2004, Artacho y Pinochet 2008).

\section{Acumulación de nutrientes}

Los valores de concentración y la absorción total de cada nutrimento efectuada por las 3 variedades de lirio en estudio durante el ciclo de crecimiento se presentan en el Cuadro 3.

El nutrimento que más absorbieron las 3 variedades de lirios (tanto por la parte aérea como por el bulbo) fue el $\mathrm{K}$, seguido en orden decreciente por el $\mathrm{N}, \mathrm{Ca}, \mathrm{P}, \mathrm{Mg}$ y $\mathrm{S}$, con excepción de Brunello el cual requirió más $\mathrm{Mg}$ que P (Cuadro 3). Según Fageria (2001), adecuados niveles de K conllevan a un uso más eficiente del $\mathrm{N}$ por las plantas, lo cual es importante debido al papel fundamental que juega el $\mathrm{N}$ en el metabolismo de las plantas y en el crecimiento de las mimas. Por otro lado, el K presenta una reacción antagonista con el $\mathrm{Ca}$ y $\mathrm{Mg}$, por lo tanto al haber mayor concentración de $\mathrm{K}$, hay menor absorción de Ca y Mg (Fageria 2001).

En cuanto a los elementos menores (Cuadro 3), en las 3 variedades, el Fe siempre presentó los valores mayores. Estos altos valores pueden deberse al elemento estructural presente en los residuos de suelo que quedaron en los bulbos y raíces, aún después del lavado de los mismos, ya que es difícil su remoción total. Los valores de $\mathrm{Zn}, \mathrm{B}$ y Mn fueron muy similares tanto en la parte aérea como en el bulbo y el $\mathrm{Cu}$, fue el elemento menos absorbido.

Por otro lado, los resultados muestran que Brunello fue la variedad que más nutrimentos absorbió, seguida en orden decreciente por Algarve y Alma Ata. Este orden de absorción de nutrimentos posiblemente se deba a que plantas pertenecientes a los grupos Asiático e Híbrido L/A (Brunello y Algarve) presentaron una mayor altura y peso que las plantas del grupo Oriental (Alma Ata) (Cuadro 3).

Es importante notar que, al igual que lo sucedido con el peso seco (Figura 1), con el paso de las semanas, la absorción de los nutrimentos por la parte aérea fue en general aumentando, mientras que en los bulbos sucedió lo contrario (Cuadro 3), lo cual concuerda con lo encontrado por Ortega et al. (2006) con los cultivares Miami, Fangio y Navona, en donde los bulbos presentaron una disminución en el contenido de nutrimentos conforme el paso de las semanas.

Lo anterior se debe a que los bulbos de las plantas madres tienen la capacidad de sustentar el crecimiento de las mismas, hasta que se presente un adecuado desarrollo de la parte aérea que le permita obtenerlos por otros medios (Miller, citado por Correa 2003). Una vez que la planta empieza a desarrollar hojas, éstas se convierten en el principal sumidero de carbohidratos. Cuando las hojas obtienen la mitad de su expansión comienzan rápidamente a reducir su importación de carbohidratos, hasta convertirse en fuente (Villalobos 2001), y posteriormente las flores pasaron a ser el sumidero más importante (Soriano 2000).

\section{Curvas de consumo real de nutrimentos}

Debido a que el aporte del bulbo es tan significativo dentro del total, se decidió representar la "absorción real" de las plantas evaluadas en la Figura 2, deduciéndole a la parte área el aporte del bulbo.

Según se observa en la Figura 2, la variedad Brunello presentó los mayores incrementos de "absorción real" de N, P y K en la última semana de su ciclo, y esta cantidad fue cercana al $60 \%$ del total consumido. En el caso de Algarve este fuerte incremento (superior a 50\%) ocurrió en la penúltima semana para el $\mathrm{N}$ y el $\mathrm{K}$ y en la última para el P (Figura 2).

La variedad Alma Ata se caracterizó por distribuir su absorción en 2 picos principales, cada uno con más del $40 \%$ de la absorción real, ubicado en las semanas 8 y 12 , especialmente para P y K. En el caso del N la absorción fue gradual, e igualmente a las otras variedades concentró su acúmulo (más del 55\%) en una sola semana correspondiente con la penúltima (12) (Figura 2).

De lo anterior, es importante resaltar 2 puntos, el primero es que un mismo elemento puede ser absorbido en momentos diferentes por 
Cuadro 3. Peso seco, concentración y absorción de elementos mayores y menores por planta, por la parte aérea y bulbo, en 3 diferentes variedades de lirios, durante su ciclo de crecimiento, en San José de la Montaña, Heredia.

\begin{tabular}{|c|c|c|c|c|c|c|c|c|c|c|c|c|c|c|c|c|c|c|c|c|c|c|c|c|}
\hline \multirow[b]{3}{*}{ Tejido } & \multirow[b]{3}{*}{ Sem } & \multirow{3}{*}{$\begin{array}{l}\text { Peso } \\
\text { seco } \\
\text { g.pl }{ }^{-1}\end{array}$} & \multicolumn{11}{|c|}{ Concentración } & \multicolumn{11}{|c|}{ Cantidad absorbida } \\
\hline & & & \multicolumn{6}{|c|}{$\%$} & \multicolumn{5}{|c|}{$\mathrm{mg} \cdot \mathrm{kg}^{-1}$} & \multicolumn{6}{|c|}{ mg.pl ${ }^{-1}$} & \multicolumn{5}{|c|}{$\mathrm{mg} \cdot \mathrm{pl}^{-1}$} \\
\hline & & & $\mathrm{N}$ & $P$ & K & $\mathrm{Mg}$ & $\mathrm{Ca}$ & S & $\mathrm{Zn}$ & B & $\mathrm{Mn}$ & $\mathrm{Fe}$ & $\mathrm{Cu}$ & $\mathrm{N}$ & $P$ & K & $\mathrm{Mg}$ & $\mathrm{Ca}$ & S & $\mathrm{Zn}$ & B & $\mathrm{Mn}$ & $\mathrm{Fe}$ & $\mathrm{Cu}$ \\
\hline \multirow{9}{*}{ 泀 } & 4 & 3,8 & 2,8 & 0,4 & 4,5 & 0,2 & 0,2 & 0,2 & 38 & 23 & 30 & 67 & 8 & 108,5 & 14,2 & 174,0 & 6,9 & 9,2 & 7,7 & 0,15 & 0,09 & 0,12 & 0,26 & 0,03 \\
\hline & 6 & 5,4 & 1,6 & 0,2 & 3,9 & 0,2 & 0,3 & 0,1 & 18 & 17 & 15 & 32 & 7 & 84,8 & 9,2 & 207,9 & 8,6 & 16,2 & 5,9 & 0,10 & 0,09 & 0,08 & 0,17 & 0,04 \\
\hline & 8 & 8,8 & 1,8 & 0,2 & 3,6 & 0,2 & 0,5 & 0,1 & 24 & 25 & 19 & 68 & 4 & 155,2 & 14,0 & 311,2 & 16,7 & 40,3 & 8,8 & 0,21 & 0,22 & 0,17 & 0,60 & 0,04 \\
\hline & 10 & 12,6 & 2,0 & 0,2 & 4,5 & 0,2 & 0,6 & 0,1 & 36 & 38 & 36 & 131 & 7 & 250,1 & 22,6 & 559,2 & 30,2 & 70,4 & 16,3 & 0,45 & 0,48 & 0,45 & 1,65 & 0,09 \\
\hline & 0 & 8,9 & 1,8 & 0,2 & 1,8 & 0,1 & 0,1 & 0,1 & 27 & 10 & 4 & 144 & 7 & 157,2 & 18,5 & 160,6 & 6,0 & 6,5 & 9,2 & 0,24 & 0,08 & 0,04 & 1,29 & 0,06 \\
\hline & 4 & 4,5 & 1,2 & 0,2 & 1,5 & 0,1 & 0,3 & 0,1 & 23 & 24 & 88 & 3805 & 16 & 51,8 & 8,0 & 65,2 & 5,8 & 14,7 & 4,9 & 0,10 & 0,11 & 0,39 & 17,00 & 0,07 \\
\hline & 6 & 3,0 & 0,8 & 0,1 & 1,3 & 0,1 & 0,4 & 0,1 & 21 & 36 & 108 & 9586 & 17 & 25,5 & 4,2 & 40,0 & 3,9 & 12,7 & 2,4 & 0,06 & 0,11 & 0,33 & 29,08 & 0,05 \\
\hline & 8 & 3,0 & 1,0 & 0,2 & 1,4 & 0,2 & 0,4 & 0,1 & 21 & 23 & 70 & 3654 & 12 & 30,0 & 5,0 & 40,6 & 4,5 & 10,4 & 3,0 & 0,06 & 0,07 & 0,21 & 10,84 & 0,04 \\
\hline & 10 & 2,5 & 1,2 & 0,2 & 1,8 & 0,2 & 0,3 & 0,1 & 25 & 23 & 69 & 3927 & 16 & 29,1 & 3,7 & 44,4 & 3,7 & 7,4 & 3,0 & 0,06 & 0,06 & 0,17 & 9,69 & 0,04 \\
\hline \multirow{9}{*}{ 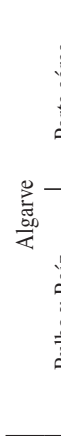 } & 4 & 2,0 & 3,2 & 0,4 & 4,8 & 0,3 & 0,4 & 0 , & 56 & 23 & 42 & 87 & 40 & 04 & 8,9 & 97,4 & 5,1 & 7,1 & 3,7 & 11 & 0,05 & 0,09 & 0,18 & 08 \\
\hline & 6 & 5,6 & 2,4 & 0,3 & 4,4 & 0,3 & 0,5 & 0 , & 38 & 22 & 28 & 66 & 11 & 132,9 & 14,6 & 249,6 & 14,1 & 28,2 & 7,3 & 21 & 0,12 & 0,16 & 0,37 & 0,06 \\
\hline & 8 & 9,1 & 2,3 & 0,2 & 5,0 & 0,3 & 0,6 & 0,1 & 39 & 30 & 39 & 76 & 5 & 212,8 & 19,2 & 456,7 & 31,1 & 53,9 & 11,9 & 0,36 & 0,27 & 0,36 & 0,69 & 0,05 \\
\hline & 10 & 10,4 & 2,2 & 0,2 & 4,1 & 0,3 & 0,6 & 0,1 & 38 & 33 & 28 & 79 & 4 & 228,8 & 20,8 & 426,4 & 35,4 & 58,2 & 12,5 & 0,40 & 0,34 & 0,29 & 0,82 & 0,04 \\
\hline & 0 & 10,9 & 1,3 & 0,3 & 1,5 & 0,1 & 0,1 & 0,1 & 35 & 6 & 6 & 64 & 7 & 144,7 & 28,1 & 161,3 & 6,3 & 5,5 & 9,0 & 0,38 & 0,07 & 0,06 & 0,69 & 0,07 \\
\hline & 4 & 6,3 & 1,1 & 0,2 & 1,8 & 0,1 & 0,2 & 0,1 & 36 & 21 & 65 & 4232 & 15 & 67,1 & 14,4 & 110,9 & 7,5 & 15,0 & 5,6 & 0,23 & 0,13 & 0,41 & 26,52 & 0,09 \\
\hline & 6 & 4,6 & 1,0 & 0,2 & 1,6 & 0,1 & 0,3 & 0,1 & 37 & 26 & 93 & 6434 & 18 & 48,2 & 9,7 & 73,7 & 5,6 & 13,0 & 4,6 & 0,17 & 0,12 & 0,43 & 29,81 & 0,08 \\
\hline & 8 & 3,8 & 1,1 & 0,1 & 2,0 & 0,1 & 0,3 & 0,1 & 26 & 17 & 58 & 3170 & 12 & 41,1 & 4,9 & 73,5 & 4,9 & 9,8 & 3,0 & 0,10 & 0,06 & 0,22 & 11,94 & 0,05 \\
\hline & 10 & 3,8 & 1,3 & 0,2 & 2,0 & 0,1 & 0,2 & 0,1 & 33 & 12 & 25 & 969 & 7 & 49,4 & 8,0 & 75,6 & 4,2 & 7,6 & 2,7 & 0,13 & 0,05 & 0,10 & 3,68 & 0,03 \\
\hline \multirow{13}{*}{ 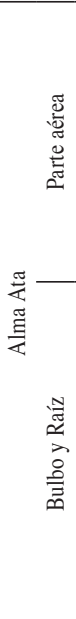 } & 4 & 1,8 & 3,7 & 0,4 & 3,7 & 0,2 & 0,1 & 0,2 & 49 & 11 & 24 & 72 & 14 & 67,3 & 6,4 & 67,7 & 2,9 & 2,2 & 3,3 & 0,09 & 0,02 & 0,04 & 0,13 & 0,03 \\
\hline & 6 & 4,7 & 2,5 & 0,2 & 2,6 & 0,2 & 0,3 & 0,1 & 25 & 10 & 22 & 100 & 4 & 116,1 & 7,1 & 120,3 & 7,5 & 12,7 & 3,8 & 0,12 & 0,05 & 0,10 & 0,47 & 0,02 \\
\hline & 8 & 6,6 & 2,2 & 0,2 & 3,9 & 0,3 & 0,6 & 0,1 & 45 & 17 & 48 & 300 & 10 & 143,9 & 12,6 & 260,0 & 19,2 & 36,5 & 7,3 & 0,30 & 0,11 & 0,32 & 1,99 & 0,07 \\
\hline & 10 & 7,9 & 1,6 & 0,2 & 3,5 & 0,2 & 0,5 & 0,1 & 36 & 17 & 29 & 253 & 8 & 124,8 & 11,9 & 272,6 & 18,2 & 39,5 & 7,1 & 0,28 & 0,13 & 0,23 & 2,00 & 0,06 \\
\hline & 12 & 11,3 & 2,0 & 0,2 & 3,6 & 0,3 & 0,5 & 0 , & 40 & 16 & 46 & 50 & 15 & 227,6 & 18,0 & 404,5 & 28,2 & 57,5 & 12,4 & 15 & 0,18 & 0,52 & 5,64 & 0,17 \\
\hline & 14 & 10,8 & 1,8 & 0,2 & 3,5 & 0,2 & 0,5 & 0,1 & 46 & 22 & 45 & 534 & 14 & 193,3 & 18,4 & 374,8 & 21,6 & 51,8 & 10,8 & 0,50 & 0,24 & 0,49 & 5,77 & 0,15 \\
\hline & 0 & 11,6 & 1,5 & 0,2 & 1,5 & 0,1 & 0,1 & 0,1 & 28 & 6 & 4 & 35 & 3 & 178,3 & 19,1 & 173,6 & 6,9 & 7,2 & 10,1 & 0,32 & 0,07 & 0,04 & 0,40 & 0,04 \\
\hline & 4 & 4,3 & 1,5 & 0,1 & 1,2 & 0,1 & 0,1 & 0,1 & 23 & 11 & 38 & 1050 & 1 & 0,1 & 5,1 & 52,9 & 3,4 & 4,3 & 3,0 & 0,10 & 0,05 & 0,16 & 4,48 & 0,03 \\
\hline & 6 & 7,2 & 0,9 & 0,1 & 1,3 & 0,1 & 0,2 & 0,1 & 28 & 20 & 141 & 4610 & 21 & 61,2 & 9,4 & 90,7 & 9,4 & 13,0 & 5,8 & 0,20 & 0,14 & 1,02 & 33,19 & 0,15 \\
\hline & & 6,0 & 0,9 & 0,1 & 1,2 & 0,1 & 0,1 & 0,1 & 17 & 12 & 58 & 1745 & 8 & 54,3 & 7,2 & 73,6 & 6,6 & 7,8 & 3,6 & 0,10 & 0,07 & 0,35 & 10,53 & 0,05 \\
\hline & 10 & 5,2 & 0,8 & 0,1 & 0,9 & 0,1 & 0,1 & 0,0 & 11 & 9 & 41 & 1243 & 7 & 41,3 & 3,7 & 46,1 & 4,2 & 6,8 & 2,1 & 0,06 & 0,05 & 0,21 & 6,51 & 0,04 \\
\hline & 12 & 6,1 & 1,0 & 0,1 & 1,4 & 0,1 & 0,2 & 0,1 & 23 & 16 & 173 & 4384 & 39 & 59,2 & 6,1 & 84,2 & 6,7 & 9,8 & 4,3 & 0,14 & 0,10 & 1,06 & 26,74 & 0,24 \\
\hline & 14 & 5,3 & 1,0 & 0,1 & 1,4 & 0,1 & 0,2 & 0,1 & 39 & 25 & 273 & 7532 & 30 & 53,7 & 6,8 & 71,6 & 7,4 & 12,1 & 4,7 & 0,21 & 0,13 & 1,44 & 39,67 & 0,16 \\
\hline
\end{tabular}



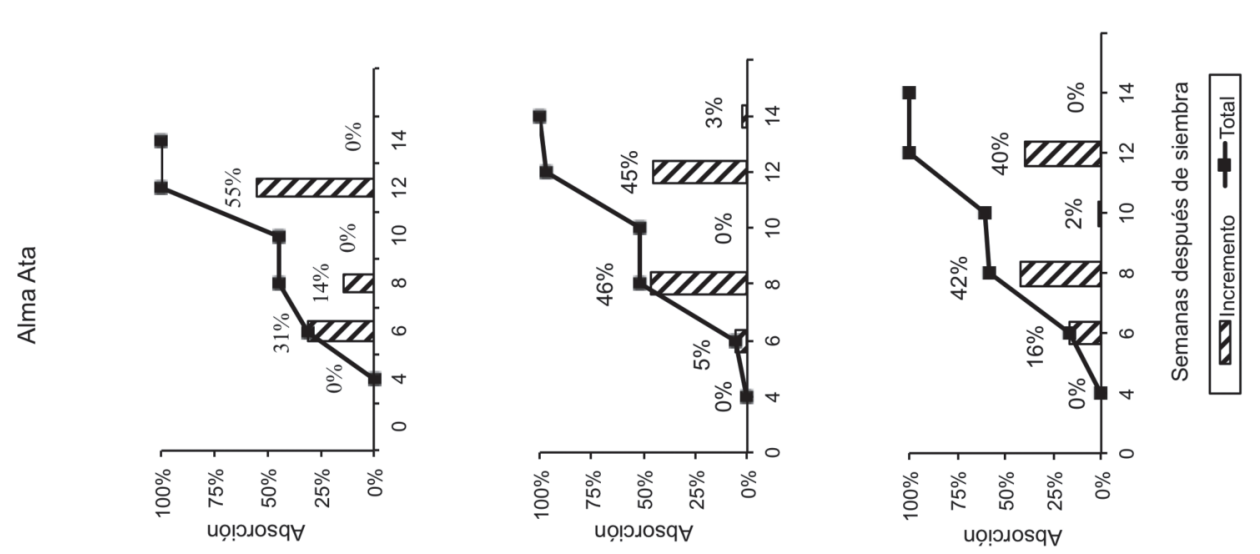

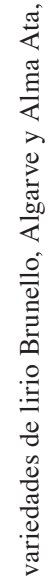
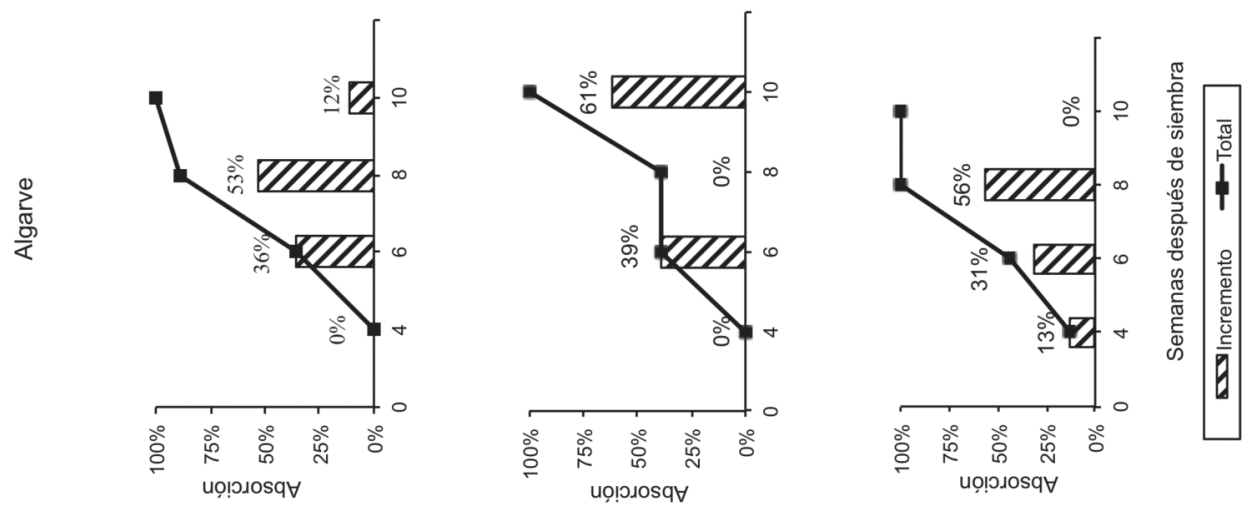

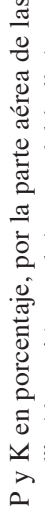
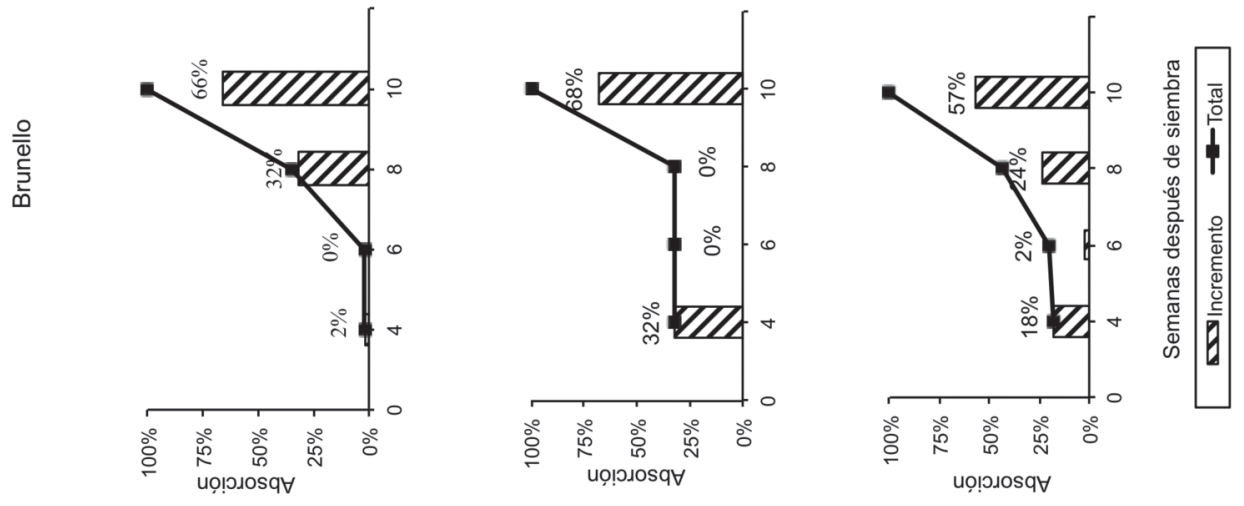

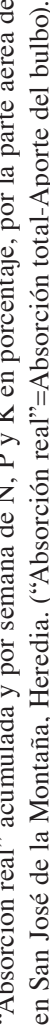

2

id 
distintas variedades de lirios, lo cual refuerza la importancia de realizar estudios para las diferentes variedades de lirios existentes y concuerda con los resultados de estudios realizados por la Universidad de Michigan State, en donde determinaron que para realizar un adecuado programa de fertilización, se deben evaluar las plantas durante todo su ciclo de crecimiento y cuantificar la cantidad de nutrimentos absorbidos por las mismas (Ortega et al. 2006).

El segundo punto es que la mayor absorción de $\mathrm{N}, \mathrm{P}$ y $\mathrm{K}$ ocurre cerca del punto de cosecha (de la semana 8 en adelante), lo cual se asemeja a los resultados obtenidos por Correa (2003), con el cultivar Miami (Oriental). Lo que sucede es que en este momento las flores se encuentran en el proceso de llenado y por lo tanto necesitan translocar todos los nutrimentos disponibles hacia dicho tejido (Bañón et al., citado por Correa 2003).

\section{Balance nutrimental de variedades de lirios}

Los requisitos de las plantas de lirio Brunello, Algarve y Alma Ata para obtener rendimientos de 72, 72 y 66 tallos. $\mathrm{m}^{-2}$ respectivamente, así como las dosis que la empresa Flor Bella S.A. aplicaba por $\mathrm{m}^{2}$ de cada uno de los elementos en el momento de este estudio, y el porcentaje de eficiencia de dicha aplicación, se muestran en el Cuadro 4.

Como se puede observar, si se expresan en términos de g. $\mathrm{m}^{-2}$, esto es a partir de la densidad de siembra de cada una, las variedades estudiadas muestran valores de requisitos de nutrimentos totales muy semejantes, a pesar de pertenecer a grupos distintos.

En cuanto a la dosis, para la mayoría de los nutrimentos, la empresa Flor Bella S.A aplicaba menos de lo requerido por las plantas. Para algunos elementos, en las variedades Brunello y Algarve no se aplicaba ni la mitad del requisito

Cuadro 4. Comparación de los "requisitos reales" de nutrimentos $\left(\mathrm{g} \cdot \mathrm{m}^{-2}\right)$ de las plantas de lirio Brunello, Algarve y Alma Ata para los rendimientos esperados de un tallo por bulbo sembrado, contra la dosis de esos nutrimentos aplicados por la finca Flor Bella S.A.

\begin{tabular}{|c|c|c|c|c|c|c|c|}
\hline & & $\mathrm{N}$ & $\mathrm{P}_{2} \mathrm{O}_{5}$ & $\mathrm{~K}_{2} \mathrm{O}$ & $\mathrm{MgO}$ & $\mathrm{CaO}$ & $\mathrm{S}$ \\
\hline \multirow[t]{3}{*}{ A-Brunello } & Requisitos de 72 tallos, g.m ${ }^{-2}$ & 10,4 & 1,9 & 37,9 & 3,30 & 6,5 & 0,90 \\
\hline & Dosis aplicada en finca, g.m $\mathrm{m}^{-2}$ & 7,9 & 12,2 & 6,1 & 0,02 & 3,2 & 0,01 \\
\hline & $\%$ Eficiencia & $131 \%$ & $16 \%$ & $622 \%$ & $19958 \%$ & $205 \%$ & $12361 \%$ \\
\hline \multirow[t]{3}{*}{ L/A-Algarve } & Requisitos de 72 tallos, g.m ${ }^{-2}$ & 10,0 & 0,4 & 31,9 & 3,98 & 5,9 & 0,44 \\
\hline & Dosis aplicada en finca, g.m $\mathrm{m}^{-2}$ & 7,9 & 12,2 & 6,1 & 0,02 & 3,2 & 0,01 \\
\hline & $\%$ Eficiencia & $125 \%$ & $4 \%$ & $523 \%$ & $23756 \%$ & $185 \%$ & $6287 \%$ \\
\hline \multirow[t]{3}{*}{ O-Alma Ata } & Requisitos de 66 tallos, g.m $\mathrm{m}^{-2}$ & 10,0 & 1,8 & 26,1 & 3,14 & 5,1 & 0,57 \\
\hline & Dosis aplicada en finca, g.m $\mathrm{m}^{-2}$ & 9,7 & 12,8 & 6,8 & 2,21 & 4,2 & 1,32 \\
\hline & $\%$ Eficiencia & $103 \%$ & $14 \%$ & $386 \%$ & $142 \%$ & $123 \%$ & $43 \%$ \\
\hline
\end{tabular}

"Requisito real": Requisito total-Aporte del bulbo.

A-Asiático; L/A-híbrido entre Lilium longiflorum x Asiático; O-Oriental.

$\%$ Eficiencia: Requisitos (g.m $\left.\mathrm{m}^{-2}\right) /$ Dosis $\left(\mathrm{g} \cdot \mathrm{m}^{-2}\right)$. 
de las plantas, como es el caso del $\mathrm{K}_{2} \mathrm{O}, \mathrm{MgO}$ y $\mathrm{S}$, que como resultado presenta porcentajes de eficiencia de los nutrimentos muy por encima del $100 \%$ (Cuadro 4).

En el caso de la variedad Alma Ata, la cantidad de $\mathrm{MgO}$ aplicado era un poco más de la mitad del requisito y el $\mathrm{S}$ aplicado resultaba más del requerido por las plantas. Debido a que es conocido que las plantas pertenecientes al grupo Oriental requieren mayor cantidad de $\mathrm{Mg}$, la finca les aplicaba específicamente a las variedades de este grupo, un suplemento adicional de Sulfato de Mg y Magnesamón.

Por el contrario, las aplicaciones de $\mathrm{P}$ por la empresa eran aproximadamente 5 veces más altas de lo requerido por las plantas de las 3 variedades de lirio (Cuadro 4). Esto quizás se deba a que la deficiencia de $\mathrm{P}$ puede llegar a ser una gran limitante en el rendimiento de cultivos (Fageria et al. Hodgson et al. Robson y Pitman citados por Fageria 2001), por lo que la empresa no quiso arriesgarse y prefirió aumentar la dosis de este elemento.

\section{Mejora del Programa de Fertilización}

Para realizar la validación de los programas de fertilización propuestos como tratamientos, se trabajó con solo una variedad, Alma Ata, por ser la más sensible a déficit nutricional; no obstante, al momento de realizar el ensayo la empresa no contaba con bulbos de Alma Ata, por lo que se dispuso trabajar con bulbos de la variedad Siberia, calibre 14/16. La variedad Siberia es también un tipo de lirio Oriental, cuyas plantas presentan un comportamiento similar a las de la variedad Alma Ata, solo que el peso seco por planta es un $20 \%$ más alto. Por esta razón, se usaron como requisitos base para el programa las necesidades de Alma Ata más un 20\%.

Los programas de fertilización Finca, Requisitos y Ajustado, expresados en términos de $\mathrm{N}, \mathrm{P}_{2} \mathrm{O}_{5}, \mathrm{~K}_{2} \mathrm{O}, \mathrm{MgO}, \mathrm{CaO}$ y $\mathrm{S}$ de cada uno de los fertilizantes granulares utilizados, se detallan en el Cuadro 5.

Cuadro 5. Cantidad de $\mathrm{N}, \mathrm{P}_{2} \mathrm{O}_{5}, \mathrm{~K}_{2} \mathrm{O}, \mathrm{MgO}, \mathrm{CaO}$ y S de cada uno de los fertilizantes granulares utilizados en los programas de fertilización Finca, Requisitos y Ajustado, en la variedad Siberia, en San José de la Montaña, Heredia.

\begin{tabular}{|c|c|c|c|c|c|c|c|c|}
\hline \multirow[t]{2}{*}{ Programa } & \multirow[t]{2}{*}{ Producto } & Cantidad & $\mathrm{N}$ & $\mathrm{P}_{2} \mathrm{O}_{5}$ & $\mathrm{~K}_{2} \mathrm{O}$ & $\mathrm{MgO}$ & $\mathrm{CaO}$ & $\mathrm{S}$ \\
\hline & & \multicolumn{7}{|c|}{ g.m $\mathrm{m}^{-2}$} \\
\hline \multirow[t]{5}{*}{ Finca } & Fórmula 12-24-12 & 50 & 6 & 12 & 6 & & & \\
\hline & Nitrato de Calcio $(15-0-0 ; 26 \% \mathrm{CaO})$ & 12 & 1,8 & 0 & 0 & 0 & 3,1 & \\
\hline & Magnesamón (21-0-0-7,5; 11\% CaO) & 6 & 1,3 & & & 0,5 & 0,7 & \\
\hline & Sulfato de Magnesio $(0-0-0-17 ; 14 \% \mathrm{~S})$ & 10 & & & & 1,7 & & 1,4 \\
\hline & & Total & 9,1 & 12,0 & 6,0 & 2,2 & 3,8 & 1,4 \\
\hline \multirow[t]{5}{*}{ Requisitos } & Fórmula 12-24-12 & 9 & 1,1 & 2,2 & 1,1 & & & \\
\hline & Nitrato de Calcio $(15-0-0 ; 26 \% \mathrm{CaO})$ & 23 & 3,4 & & & & 5,9 & \\
\hline & Nitrato de Potasio (13-0-44) & 59 & 7,6 & & 25,9 & & & \\
\hline & KMag $(0-0-22-18 ; 22 \% \mathrm{~S})$ & 20 & & & 4,5 & 3,7 & & 4,5 \\
\hline & & Total & 12,1 & 2,2 & 31,4 & 3,7 & 5,9 & 4,5 \\
\hline \multirow[t]{6}{*}{ Ajustado } & DAP (18-46-0) & 14 & 2,6 & 6,6 & 0 & & & \\
\hline & Nitrato de Calcio $(15-0-0 ; 26 \% \mathrm{CaO})$ & 12 & 1,8 & & & & 3,1 & \\
\hline & Nitrato de Potasio (13-0-44) & 12 & 1,6 & & 5,3 & & & \\
\hline & Sulfato de Potasio $(0-0-22-18 ; 22 \% \mathrm{~S})$ & 16 & & & 8,1 & & & 2,8 \\
\hline & KMag $(0-0-22-18 ; 22 \% \mathrm{~S})$ & 11 & 0 & & 2,4 & 1,9 & & 2,4 \\
\hline & & Total & 6,0 & 6,6 & 15,8 & 1,9 & 3,1 & 5,1 \\
\hline
\end{tabular}




\section{Respuesta de las plantas de lirio variedad Siberia a los programas}

\section{Tamaño de las plantas}

El Cuadro 6 muestra el número de plantas, peso fresco y número de botones totales por $\mathrm{m}^{2}$, obtenidos al separar por tamaños las plantas de los diferentes programas de fertilización evaluados.

Como es posible observar, las plantas de tamaño grande no mostraron diferencias significativas entre los diferentes programas, lo cual sí sucedió con las plantas de tamaño mediano y pequeño.

En el caso de las plantas de tamaño pequeño, la gran diferencia significativa se presentó entre los 4 programas y el Testigo Absoluto, lo cual demuestra la importancia de la fertilización. Excluyendo del análisis al Testigo Absoluto, los programas en los que el número de plantas pequeñas, que probablemente ofrecieron brotes de menor calidad, fue más abundante (aunque no significativo estadísticamente) fueron el Sin Granular y el Ajustado, lo que parece indicar que bajar tanto la dosis (hasta la mitad del requisito, excepto en el caso del P, que fue lo que se hizo en el Ajustado) no permite mantener la calidad de los tallos finales obtenidos.

Es importante resaltar la semejanza, estadísticamente significativa, que se presentó entre los programas Finca y Requisitos en todas las variables evaluadas (número de plantas, peso y número de botones presentes en cada tipo de tamaño de planta) a pesar de las diferencias entre las cantidades de nutrimentos aplicados en cada programa: en el programa Requisitos, la dosis aplicada fue de casi una sexta parte del P que se aplica regularmente en la finca. Esto demuestra que un elemento como el $\mathrm{P}$, se adicionaba en la Finca, durante su rutina regular en cantidades muy elevadas e innecesarias.

Cuadro 6. Promedio del número de plantas, peso fresco de plantas y número de botones por $\mathrm{m}^{2}$ en plantas de lirio variedad Siberia de tamaño pequeño $(<80 \mathrm{~cm})$, mediano $(80-90 \mathrm{~cm})$ y grande $(>90 \mathrm{~cm})$, sometidas a diferentes programas de fertilización con mitad foliar y el Testigo absoluto, en San José de la Montaña, Heredia.

\begin{tabular}{|c|c|c|c|c|c|c|c|c|c|}
\hline \multirow[b]{2}{*}{ 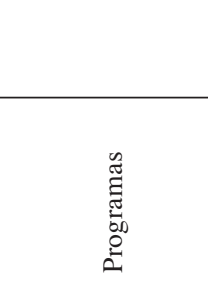 } & \multicolumn{3}{|c|}{$\begin{array}{l}\text { Número de } \\
\text { Plantas. } m^{-2}\end{array}$} & \multicolumn{3}{|c|}{$\begin{array}{l}\text { Peso Fresco de } \\
\text { Plantas. } \mathrm{m}^{-2}\end{array}$} & \multicolumn{3}{|c|}{$\begin{array}{l}\text { Número de Botones en } \\
\text { Plantas. } \mathrm{m}^{-2}\end{array}$} \\
\hline & 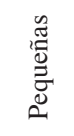 & 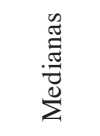 & $\frac{\tilde{v}}{\tilde{E}}$ & 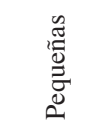 & 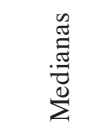 & 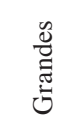 & 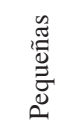 & 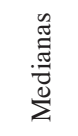 & $\frac{\tilde{U}}{\tilde{E}}$ \\
\hline Finca & $17 \mathrm{a}$ & $23 \mathrm{bc}$ & $8 \mathrm{a}$ & $702 \mathrm{a}$ & $1282 \mathrm{c}$ & $635 \mathrm{a}$ & $30 \mathrm{a}$ & $61 \mathrm{~cd}$ & $30 \mathrm{a}$ \\
\hline Requisitos & $15 \mathrm{a}$ & $24 \mathrm{c}$ & $9 \mathrm{a}$ & $650 \mathrm{a}$ & $1473 \mathrm{c}$ & $688 \mathrm{a}$ & $29 \mathrm{a}$ & $65 \mathrm{~d}$ & $29 \mathrm{a}$ \\
\hline Ajustado & $23 \mathrm{a}$ & $15 \mathrm{~b}$ & $11 \mathrm{a}$ & $945 \mathrm{a}$ & $915 \mathrm{~b}$ & $762 \mathrm{a}$ & $52 \mathrm{a}$ & $42 \mathrm{~b}$ & $33 \mathrm{a}$ \\
\hline Sin Granular & $22 \mathrm{a}$ & $17 \mathrm{bc}$ & $5 \mathrm{a}$ & $783 \mathrm{a}$ & $903 \mathrm{bc}$ & $390 \mathrm{a}$ & $52 \mathrm{a}$ & $43 \mathrm{bc}$ & $18 \mathrm{a}$ \\
\hline Testigo Absoluto & $39 \mathrm{~b}$ & $0 \mathrm{~b}$ & $0 \mathrm{a}$ & $1517 \mathrm{~b}$ & $0 \mathrm{~b}$ & $0 \mathrm{a}$ & $92 \mathrm{~b}$ & $0 \mathrm{~b}$ & $0 \mathrm{a}$ \\
\hline
\end{tabular}

* Letras distintas indican diferencias significativas con $\mathrm{LSD}<\mathrm{y} / \mathrm{ó}=0,05$. 
De igual manera, el hecho que el tratamiento Requisitos haya dado resultados tan parecidos al de Finca, pese a que en el de Requisitos se aplicó hasta 5 veces más K, debido a que es ampliamente requerido por las plantas de lirio, es un indicador que este elemento, cuando los niveles en el suelo son adecuados, es posible que pueda ser suplido parcialmente por este, y su aplicación, en grandes cantidades, no resulte en una ventaja significativa en términos cuantitativos de rendimiento.

Es importante notar que, según la clasificación realizada por Betancourt et al. (2005), las plantas de primera calidad son aquellas con una longitud de tallo entre 80 a $90 \mathrm{~cm}$ y las de segunda calidad tienen una longitud de 70 a $80 \mathrm{~cm}$, en este estudio se obtuvieron mayormente plantas de segunda calidad (Cuadro 6). Por otro lado al sumar las plantas de tamaño mediano y grande, se obtuvo el mayor porcentaje cuando se aplicó el programa Requisitos estrictamente (Cuadro 6), lo cual refleja que de este programa se obtuvo la mayor cantidad de plantas deseables para exportación.

Este impacto sobre la calidad que presenta el programa Requisitos, podría asociarse a ese exceso de $\mathrm{K}$ aplicado, que ofreció niveles disponibles más altos a la planta que en cualquiera de los otros programas.

\section{Producción total de botones}

La abundancia (número) y calidad (tamaño) de botones producidos por cada uno de los programas utilizados y en el Testigo Absoluto, fueron otras variables de rendimiento evaluadas. En la Figura 3 se presentan los datos en forma porcentual.

Las plantas de los programas Requisitos y Ajustado obtuvieron un mayor porcentaje de botones de tamaño grande (31\% y $28 \%$ respectivamente), con respecto al programa Finca, lo cual es importante debido a que son los botones de tamaño grande los que se espera y desea obtener (Figura 3) (Correa 2003).

Esta mejoría en los tamaños de los botones, probablemente se deba a la mayor cantidad de $\mathrm{K}, \mathrm{Mg}$ y $\mathrm{S}$ que fue aplicado a las plantas bajo el programa Requisitos y Ajustado con respecto al de Finca, ya que se ha demostrado, en otros cultivos, que estos elementos ayudan a mejorar la calidad de los mismos; asimismo pudo haber influido el sinergismo que existe entre el $\mathrm{K}$ y $\mathrm{N}$ (Lazcano 1998, IPNI 2010).
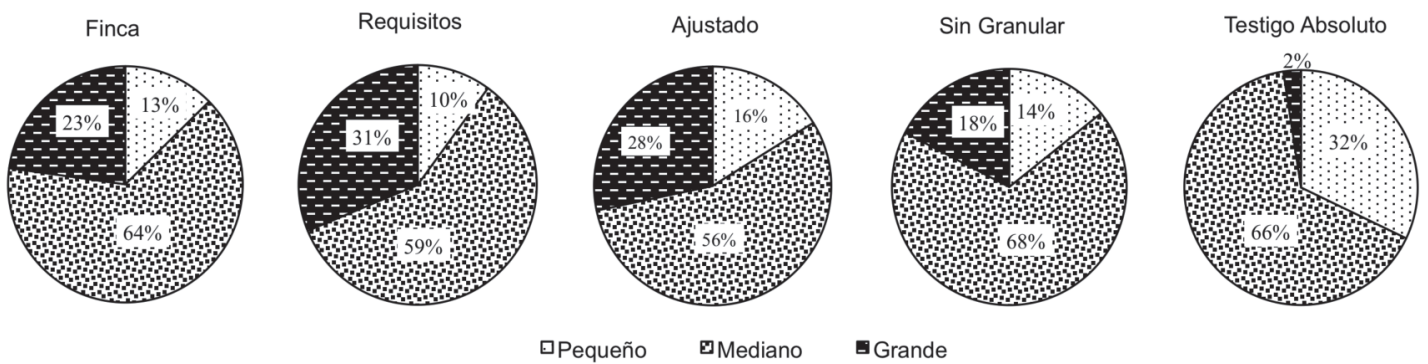

Fig. 3. Porcentaje de botones de tamaño pequeño, mediano y grande, en plantas de lirios variedad Siberia, en San José de la Montaña, Heredia, en los diferentes programas de fertilización con mitad foliar utilizados y el Testigo Absoluto. 


\section{Apariencia de los bulbos}

Finalmente, se realizó una caracterización del estado que presentaban los bulbos a la cosecha, clasificándolos según las siguientes categorías:

Categoría mala: bulbos con muy poca y mala raíz adventicia.

Categoría media: bulbos con poca, pero buena raíz adventicia.

Categoría buena: bulbos con mucha y buena raíz adventicia.

En la Figura 4, se muestra la distribución porcentual de estas categorías que se presentó en cada uno de los programas de fertilización aplicados al campo, así como en el Testigo Absoluto.

Las plantas de los programas Finca, Requisitos y Ajustado, presentaron una distribución semejante entre las distintas categorías (28 a 39\%), donde las plantas del programa Finca, obtuvieron el mayor porcentaje de bulbos con categoría mala (39\%), y las plantas del programa Requisitos y Ajustado las que mostraron mayor porcentaje de bulbos con categoría buena (35-34\%) (Figura 4). Esta distribución es muy semejante a la que presentaron los bulbos en la condición de Testigo Absoluto, lo que podría demostrar la baja influencia de la fertilización al suelo sobre el comportamiento de los bulbos.

Por el contrario, el efecto que es muy relevante de destacar, es el que presentaron los bulbos de las plantas del programa Sin Granular, ya que mostraron un significativo mayor porcentaje de bulbos de categoría buena (56\%) (Figura 4). Asimismo, el programa Sin Granular mostró la mejor apariencia de bulbos y raíces, así como la mayor cantidad de raíces adventicias, lo cual posiblemente se deba a un efecto de sobrevivencia de las plantas de lirio sometidas a este programa. Lo que probablemente sucedió fue que las raíces de lirio al no tener nutrimentos disponibles cerca de ella (porque no se les aplicó ningún fertilizante granular), empezaron a extenderse, para buscar alimento en el suelo (Roh 1999), lo cual explica la mayor cantidad de raíces con respecto a las obtenidas por las plantas de los otros programas.

Otro aspecto importante a considerar es la participación de la fertilización foliar, ya que si la sola disponibilidad en el suelo hubiera sido necesaria, se habría obtenido un comportamiento similar al programa Sin Granular con los bulbos y raíces de las plantas del Testigo Absoluto, cosa que no sucedió. Las plantas del programa Sin Granular, si bien es cierto no recibieron fertilizantes granulares, sí recibieron foliares, lo cual posiblemente produjo un efecto estimulante que propició que las raíces de estas plantas empezaran a buscar nutrimentos en el suelo para absorberlos y transportarlos a las partes aéreas, repercutiendo en un mayor crecimiento de las raíces.

Caso contrario podría haber sucedido con las plantas de los otros programas, en donde al darles los fertilizantes necesarios para su sobrevivencia, las raíces no se estimularon y no tendieron a extenderse, ni presentar la buena apariencia que presentaron las raíces de las plantas del programa Sin Granular.
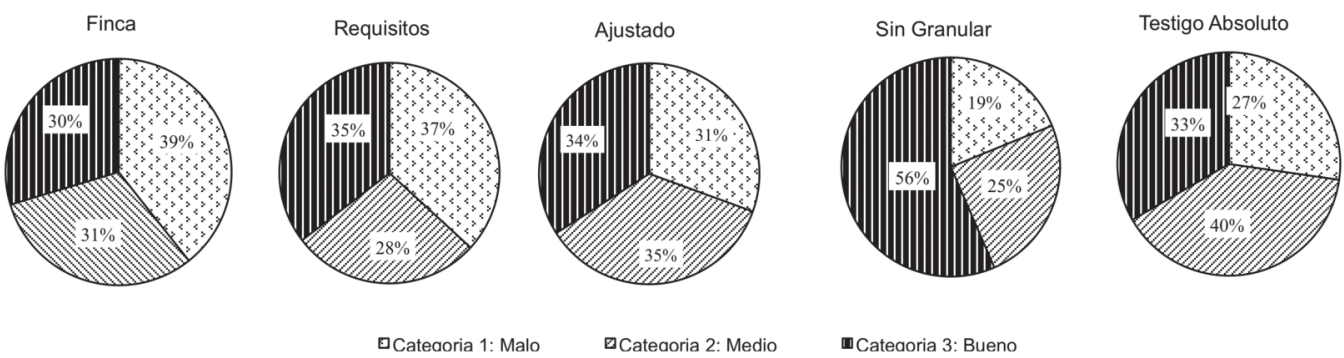

Fig. 4. Porcentaje de bulbos de la variedad de lirio Siberia, de apariencia mala, media o buena en los diferentes programas de fertilización con mitad foliar utilizados y el Testigo Absoluto, en San José de la Montaña, Heredia. 


\section{LITERATURA CITADA}

ARCE R. 2009. El mercado de flores en Estados Unidos. Promotora del Comercio Exterior de Costa Rica (PROCOMER), San José, C.R. 40 p.

ARCE R., CHACÓN E., CHAVES G., TRISTÁN A. 2008. Estadísticas de Comercio Exterior de Costa Rica. Promotora del Comercio Exterior de Costa Rica (PROCOMER), San José, C.R. 220 p.

ARTACHO P., PINOCHET D. 2008. Producción de materia seca y absorción de nitrógeno del cultivo del tulipán (Tulipa gesneriana L.). Agrociencia 42:37-45.

BERTSCH F. 2003. Absorción de nutrimentos por los cultivos. Asociación Costarricense de la Ciencia del suelo (ACCS). San José. Costa Rica. 307 p.

BETANCOURT M., RODRÍGUEZ M., SANDOVAL M., GAYTÁN E. 2005. Fertilización foliar una herramienta en el desarrollo del cultivo de Lilium cv. Stargazer. Revista Chapingo. Serie horticultura. Vol. 11 (002):371-378.

CHAVES G., CERDAS V., LÓPEZ K., TRISTAN A., VARGAS L. 2009. Estadísticas de Comercio Exterior de Costa Rica. Promotora del Comercio Exterior de Costa Rica (PROCOMER), San José, C.R. 244 p.

CORREA M. 2003. Caracterización de los estados de desarrollo y determinación de las curvas de absorción de nutrientes en Lilium sp. Cvs. Miami, Navona y Fangio para flores de corte. Tesis Mag. Sc. Santiago, Chile. Pontificia Universidad Católica de Chile. 90 p.

FACCHINETTI C., MARINANGELI P. 2008. Avances en la producción nacional de bulbos de lilium (en línea). AgroUNS 5 (9):5-9. Editorial de la Universidad Nacional del Sur. Consultado 15 mar. 2009. Disponible en http://www.criba.edu.ar/agronomia

FAGERIA V. 2001. Nutrient interactions in crop plants. Journal of Plant Nutrition 24 (8):1269-1290.
HERNÁNDEZ J. 2008. Perfil producto: plantas, flores y follajes. Promotora del Comercio Exterior de Costa Rica (PROCOMER), San José, C.R. 38 p.

INTERNACIONAL PLANT NUTRITION INSTITUTE (IPNI). 2010. Efecto del potasio, magnesio y azufre en el rendimiento y calidad de la caña de azúcar (en línea). Consultado 20 nov. 2010. Disponible en: www.ipni.net/ppiweb/mexnca.nsf/\$webindex/8A59F DE0857BAF0306256C1500680481

LAZCANO I. 1998. El papel del azufre y el potasio en la producción de hortalizas de alta calidad en México. $3(2): 1-5$

ORTEGA R., CORREA M., OLATE E. 2006. Determinación de las curvas de acumulación de nutrientes en tres cultivares de Lilium spp., para flor de corte. Agrociencia 40:77-88.

PROCOMER 2004-2008. Costa Rica: Estadísticas de Exportación 2003-2007 (en línea). Consultado: 15 nov. 2010. Disponible en: www.procomer.com

PROCOMER 2010. Costa Rica: Estadísticas de Exportación Octubre 2010. (en línea). Consultado: 15 nov. 2010. Disponible en: www.procomer.com

ROH M. 1999. Physiology and Management of Lilium bulbs. Acta Hort. 482:39-46.

SORIANO C. 2000. Efecto de la fertilización tradicional y una ajustada a la curva de extracción de nutrientes en Lilium cv. Sancerre. Taller de licenciatura. Facultad de Agronomía, Universidad Católica de Valparaíso. Quillota, Chile. 48 p.

TOIT E., ROBBERTSE P., NIEDERWIESES J. 2004. Plant carbohydrate partitioning of Lachenalia cv. Ronina during bulb production. Scientia Horticulturae 102:433-440.

VILLALOBOS R. 2001. Fisiología de la producción de los cultivos tropicales. Fascículo I: Procesos fisiológicos básicos. Editorial Universidad de Costa Rica. San José, Costa Rica. 228 p. 